\title{
Measurements of proton-induced reactions on ruthenium-96 in the ESR at GSI
}

\author{
G. Rastrepina ${ }^{* a, b}$ T. Aumann ${ }^{a}$, S. Bishop ${ }^{c}$, K. Blaum $^{d}$, K. Boretzky ${ }^{a}$, F. Bosch ${ }^{a}$, \\ H. Bräuning ${ }^{a}$, C. Brandau ${ }^{a, c}$, T. Davinson ${ }^{e}$, I. Dillmann $^{c}$, C. Dimopoulou ${ }^{a}$, \\ O. Ershova ${ }^{a, b}$, Z. Fülöp ${ }^{f}$, H. Geissel ${ }^{a}$, G. Gyürky ${ }^{f}$, M. Heil ${ }^{a}$, F. Käppeler ${ }^{h}$, \\ A. Kelić-Heil $^{a}$, C. Kozhuharov ${ }^{a}$, C. Langer ${ }^{a, b}$, T. Le Bleis ${ }^{c}$, Y.A. Litvinov ${ }^{a, d}$, G. Lotay ${ }^{e}$, \\ J. Marganiec ${ }^{a}$, G. Münzenberg ${ }^{a}$, F. Nolden ${ }^{a}$, N. Petridis ${ }^{b}$, R. Plag ${ }^{b}$, U. Popp ${ }^{a}$, \\ R. Reifarth ${ }^{b}$, B. Riese ${ }^{a}$, C. Rigollet ${ }^{i}$, C. Scheidenberger ${ }^{a}$, H. Simon ${ }^{a}$, K. Sonnabend ${ }^{b}$, \\ M. Steck ${ }^{a}$, T. Stöhlker ${ }^{a}$, K. Sümmerer ${ }^{a}$, T. Scücs ${ }^{f}$, G. Weber ${ }^{a}$, H. Weick ${ }^{a}$, \\ D.F.A. Winters ${ }^{a}$, N. Winters ${ }^{a}$, P.J. Woods ${ }^{e}$, Q. Zhong ${ }^{j}$ \\ E-mail:g.rastrepina@gsi.de \\ ${ }^{a}$ Gesellschaft für Schwerionenforschung mbH, Darmstadt, D-64291, Germany \\ ${ }^{b}$ J.W. Goethe-Universität, Frankfurt am Main, D-60438, Germany \\ ${ }^{c}$ Technische Universität München, 85748 Garching, Germany \\ ${ }^{d}$ Max-Planck-Institut für Kernphysik, 69117 Heidelberg, Germany \\ ${ }^{e}$ University of Edinburgh, Edinburgh, $U K$ \\ ${ }^{f}$ Institute of Nuclear Research, Debrecen, H-4026, Hungary \\ ${ }^{h}$ Karlsruhe Institut für Technologie, Institut für Kernphysik, 76021 Karlsruhe, Germany \\ ${ }^{i}$ Kernfysisch Versneller Instituut, NL-9747 AA Groningen, Netherlands \\ ${ }^{j}$ China Institute of Atomic Energy, 102413 Beijing, China
}

\begin{abstract}
Storage rings offer the possibility of measuring proton- and alpha-induced reactions in inverse kinematics. The combination of this approach with a radioactive beam facility allows, in principle, the determination of the respective cross sections for radioactive isotopes. Such data are highly desired for a better understanding of astrophysical nucleosynthesis processes like the p-process. A pioneering experiment has been performed at the Experimental Storage Ring (ESR) at GSI using a stable ${ }^{96} \mathrm{Ru}$ beam at $9-11 \mathrm{AMeV}$ and a hydrogen target. Monte-Carlo simulations of the experiment were made using the Geant 4 code. In these simulations, the experimental setup is described in detail and all reaction channels can be investigated. Based on the Geant 4 simulations, a prediction of the shape of different spectral components can be performed. A comparison of simulated predictions with the experimental results shows a good agreement and allows the extraction of the cross section.
\end{abstract}

8th International Conference on Nuclear Physics at Storage Rings-Stori11,

October 9-14, 2011

Laboratori Nazionale di Frascati, Italy

${ }^{*}$ Speaker. 


\section{Introduction}

Most of the elements heavier than iron are built up in stars via neutron capture in the s- or $\mathrm{r}$ processes. s-(slow)-process is the process of neutron capture with the emission of gamma radiation $(\mathrm{n}, \gamma)$ which takes place on a long time-scale with neutron densities in the order of $10^{6}$ to $10^{11} \mathrm{~cm}^{-3}$. The neutron captures occur at slow rates compared to the intervening $\beta$-decays. The s-process produces the abundance peaks at $\mathrm{A}=90,138$ and 208. The $\mathrm{r}$-(rapid)-process is the process of neutron capture on a very short time-scale with neutron densities greater than $10^{20} \mathrm{~cm}^{-3}$. The neutron captures occur then much faster than the competing beta-decays. The r-process produces the abundance peaks at $\mathrm{A}=80,130$ and 194 [1].

There are, however, 35 nuclei on the proton-rich side of the valley of stability, which cannot be produced by neutron capture, because the path of these processes are blocked by stable isotopes. These are the so-called p-nuclei, which typically have much lower natural abundances compared to their more neutron-rich stable isotopes. The proton-rich nuclei are produced by a combination of the $(\mathrm{p}, \gamma),(\gamma, \mathrm{n}),(\gamma, \mathrm{p})$ and $(\gamma, \alpha)$ reactions on existing s- or r-nuclei at stellar temperatures around a few GK, characteristic of explosive environments. To adequately describe the p-process nucleosynthesis, one needs reliable information on the thousands of reaction rates involved. Unfortunately, there is a considerable lack of experimental data on the relevant cross sections in the p-process energy range, because a direct measurement is very difficult for most $\gamma$-induced reactions [2]. To overcome this difficulty, the charged particle induced reaction cross sections are measured and their inverse photodisintegration reaction cross sections are calculated using the detailed balance theorem [3]. The p-process occurs in supernovae, where $(\mathrm{p}, \gamma)$ and $(\gamma, \mathrm{n})$ reactions occur on the seed of s- and r-nuclei at high temperatures. The p-nucleus ${ }^{96} \mathrm{Ru}$ was chosen for testing a new experimental approach to determine the proton capture cross section at astrophysical energies in inverse kinematics (Figure 1).

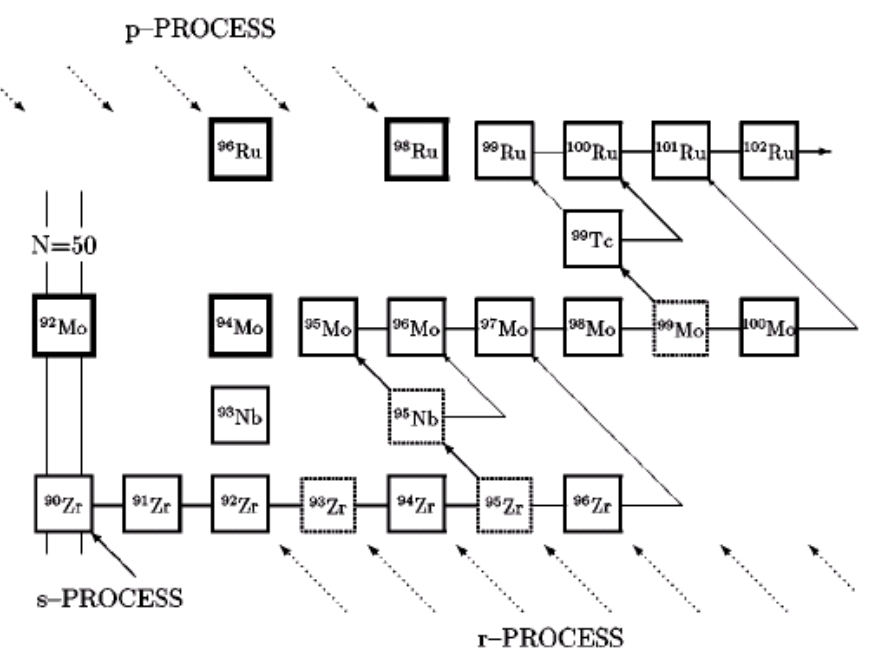

Figure 1: Synthesis of the elements in the mass region below $A=100 .{ }^{96} \mathrm{Ru}$ is shielded from s- and r-process paths by unstable resp. stable isotopes and can only be produced via p-process. 
Most of the few existing data in the Gamov window of the p-process have been obtained applying the activation technique. But this method is limited because it is only applicable if the reaction product is radioactive and the sample material is not too short-lived to produce a sample. No such limits exist for the proposed new experiments in storage rings with circulating heavy-ion beams, since the reaction products are identified by their mass-over-charge ratios and isotopes with half-lives down to a few minutes could be investigated. The purpose of this experiment was to benchmark the new approach by comparing our results with the activation method and to explore the possibilities of a new method for measuring the same reactions with short-lived isotopes and stable reaction products.

\section{Experimental technique}

A pioneering experiment has been performed at the Experimental Storage Ring (ESR) at GSI using a stable ${ }^{96} \mathrm{Ru}$ beam and a hydrogen gas target. The primary beam was sent from the UNILAC accelerator to the synchrotron SIS (Figure 2) where it was accelerated to $100 \mathrm{AMeV}$ and then stripped using a carbon foil $\left(11 \mathrm{mg} / \mathrm{cm}^{2}\right)$. Afterwards the fully stripped ions were injected into the ESR (Figure 3) and slowed down to energies of 9, 10 and $11 \mathrm{AMeV}$. After slowing down, the ruthenium ions were stored in the ESR for several minutes. The thickness of the newly developed hydrogen target was $10^{13} / \mathrm{cm}^{2}$ [4].

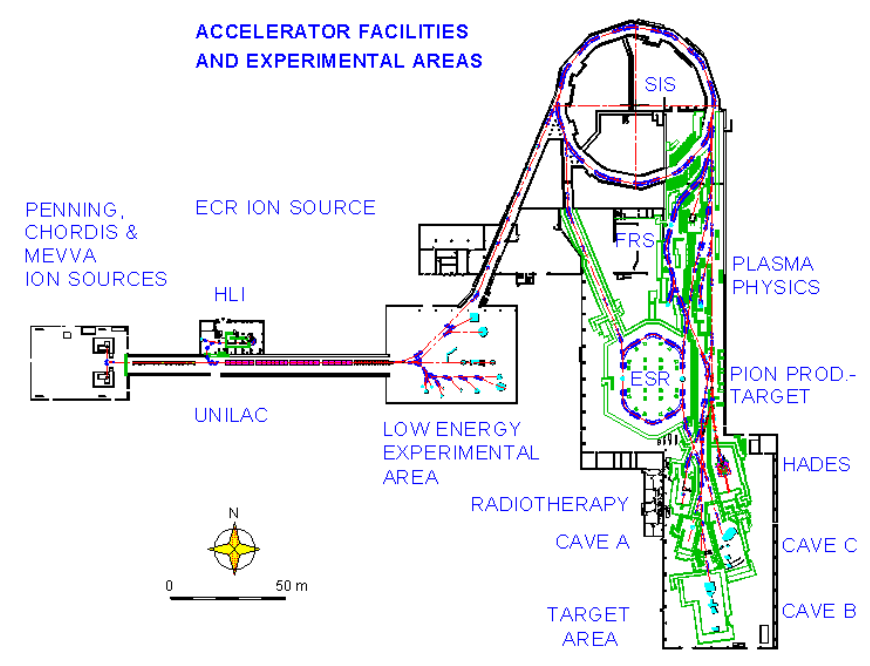

Figure 2: The accelerator complex at GSI.

The most important interactions of fully strip ruthenium ions with hydrogen molecules are electron pick-up and proton-induced nuclear reactions like $(\mathrm{p}, \gamma),(\mathrm{p}, \mathrm{n})$ and $(\mathrm{p}, \alpha)$.

Because of their different $\mathrm{A} / \mathrm{Z}$ ratio, the products of proton-induced reactions $\left({ }^{97} \mathrm{Rh}\right)$ are spatially separated from the unreacted ${ }^{96} \mathrm{Ru}$ ions after the first dipole and could be detected by two Double Sided Silicon Strip Detectors (DSSSD) mounted in a pocket on the inside of the ESR. Each detector had 16 strips in X-and Y- direction. The strip pitch was $3 \mathrm{~mm}$ and the strip length was $49.5 \mathrm{~mm}$. The detectors were arranged side by side to increase the covered area. However, due 
to connecters etc., a gap of $25 \mathrm{~mm}$ remained between both active areas. In order to measure a continous spectrum, data were taken with different detector positions, e.g. at nominal position and shifted by $25 \mathrm{~mm}$. The efficiency for (p, $\gamma$ ) events was close to $100 \%$.

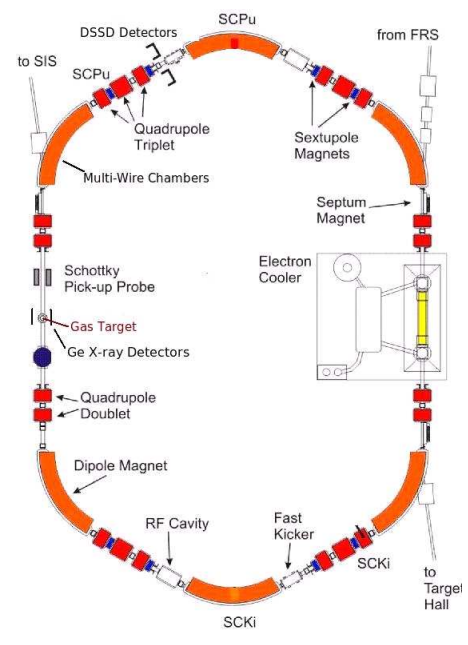

Figure 3: The Experimental Storage Ring (ESR) at GSI.

As the experiment was performed at energies of 9-11 AMeV, competing $(\mathrm{p}, \mathrm{n})$ and $(\mathrm{p}, \alpha)$ reactions occured too and they could not be neglected. These events have to be discriminated from the desired proton capture reactions based on the position on the DSSSD. An additional background component is the elastic scattering of ruthenium ions on protons.

Multi-Wire Counters were installed on the outer side of the first dipole after the hydrogen target to detect products from electron pick-up. Germanium X-ray detectors were mounted close to the target at angles 35 and 90 degree to detect the emitted X-rays.

The particle position in the DSSSD for both detector positions is shown in Figure 4. The combined spectrum for both detector positions is represented in Figure 5. Events from ${ }^{96} \mathrm{Ru}(\mathrm{p}, \gamma){ }^{97} \mathrm{Rh}$ were normalised by events from the electron capture reaction in our target.

\section{Monte-Carlo simulations with Geant 4 code}

In order to understand and subtract background from competing reactions, the most intense background sources were simulated using Geant4 [5]. The simulation comprises all beam pipes, quadrupole magnets and the dipole between the hydrogen target and the detector location as well as the DSSSD itself (Figure 6).

Based on Geant4 simulations, a prediction of the distribution of the reaction products in the detector for different reactions was performed. Figure 7 shows the results for the ${ }^{96} \mathrm{Ru}(\mathrm{p}, \gamma)$ reaction itself as well as for background from (p,n), $(\mathrm{p}, \alpha)$ and elastic scattering (p,p).

\section{Cross section determination}

The reaction cross section was measured relative to the well known ${ }^{96} \mathrm{Ru}^{44+}\left(\mathrm{e}^{-}\right){ }^{96} \mathrm{Ru}^{43+}$ cross section, thereby eliminating uncertainties from target thickness and number of primary ${ }^{96} \mathrm{Ru}$ ions. 

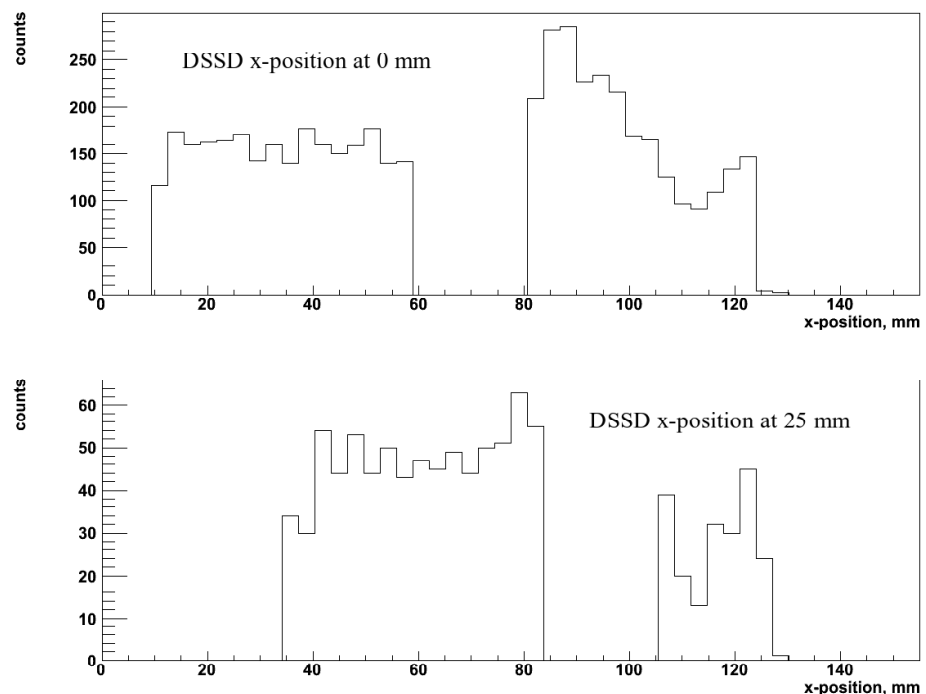

Figure 4: Position distribution of secondary particles on the DSSSDs with the detector at the nominal position (top) and shifted by $25 \mathrm{~mm}$ to the right (bottom). The region above $\mathrm{x}=125 \mathrm{~mm}$ was shielded by the beam pipe.

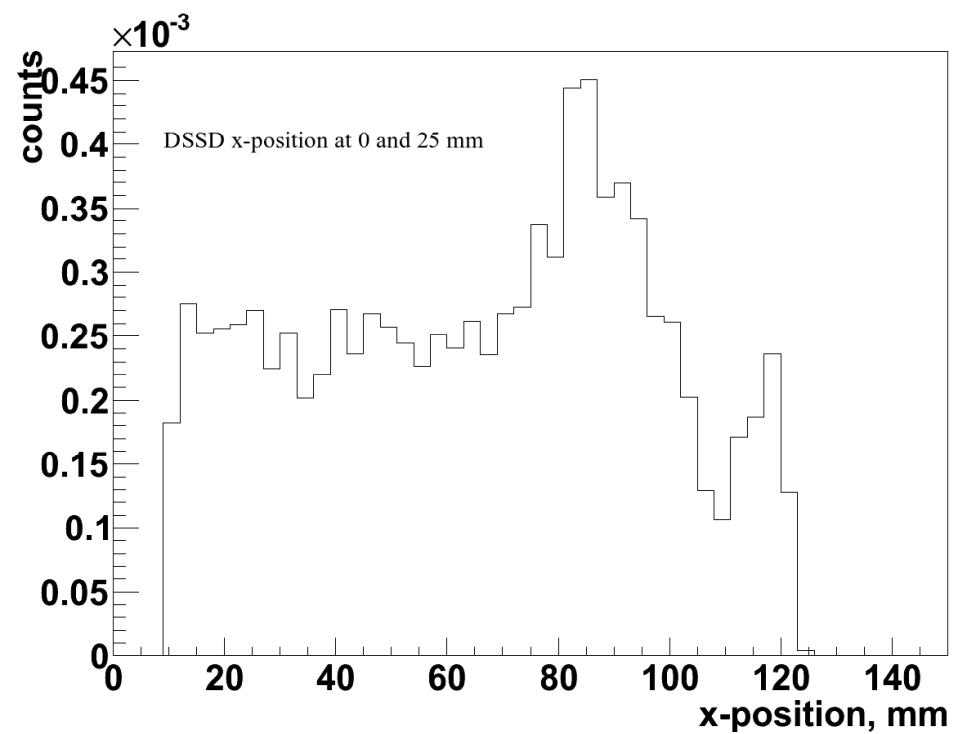

Figure 5: Combined spectrum of DSSSD for detector positions at 0 and $25 \mathrm{~mm}$. The peak of ${ }^{96} \mathrm{Ru}(\mathrm{p}, \gamma)$ is clearly visible at $\mathrm{x}=85 \mathrm{~mm}$.

If $Z_{p, \gamma}$ is the number of nuclei produced by proton captures (peak area), $\sigma_{p, \gamma}$ the proton capture cross section, $Z_{e c}$ the number of nuclei produced via electron pick-up and $\sigma_{e c}$ the electron pick-up cross section then 


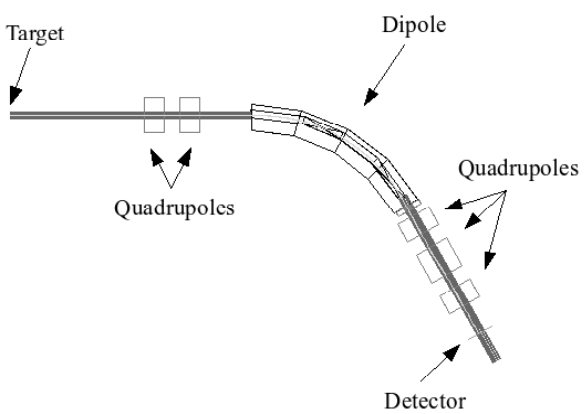

Figure 6: Geant 4 simulations of secondary particles from ${ }^{96} \mathrm{Ru}(\mathrm{p}, \gamma){ }^{97} \mathrm{Rh}$ reaction.
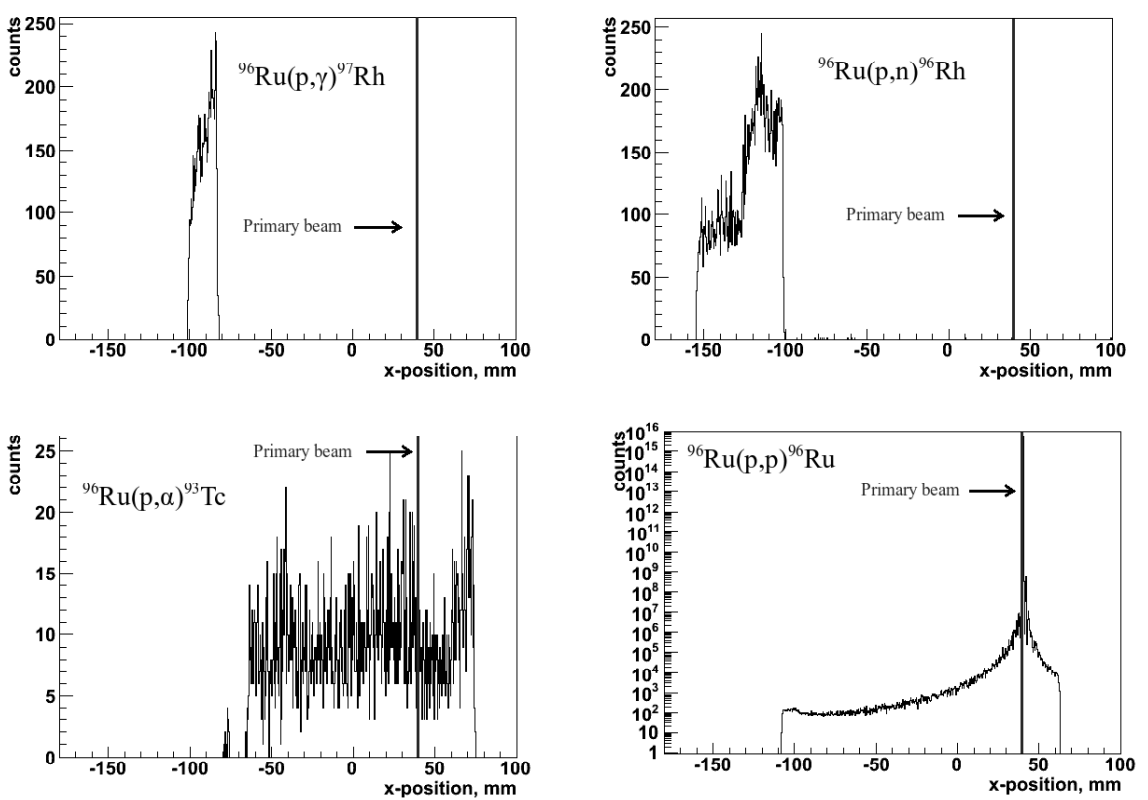

Figure 7: Simulated position distribution of reaction products from different reactions on the DSSSD.

$$
\sigma_{p, \gamma}=\sigma_{e c} \cdot \frac{Z_{p, \gamma}}{Z_{e c}}
$$

Since the atomic electron pick-up cross section is well known, the desired proton capture cross section can be determined solely based on the ratio of detected counts in the silicon detectors and in the multi-wire chamber. Based on Geant 4 simulations the background for the ${ }^{96} \mathrm{Ru}(\mathrm{p}, \gamma){ }^{97} \mathrm{Rh}$ reaction can be estimated. The important background components are ${ }^{96} \mathrm{Ru}(\mathrm{p}, \mathrm{n}){ }^{96} \mathrm{Rh},{ }^{96} \mathrm{Ru}(\mathrm{p}, \alpha){ }^{93} \mathrm{Tc}$, ${ }^{96} \mathrm{Ru}(\mathrm{p}, \mathrm{p}){ }^{96} \mathrm{Ru}$ reactions. A rough estimate of the proton capture cross section of ${ }^{96} \mathrm{Ru}$ at $11 \mathrm{AMeV}$ 
gives 1.5-3.5 mb, which is comparable to the predictions from the NON-SMOKER code [6].

\section{Summary and outlook}

A pioneering experiment was performed at the Experimental Storage Ring (ESR) at GSI. Fully stripped ions of ${ }^{96} \mathrm{Ru}$ were injected into the storage ring and slowed down to $9-11 \mathrm{MeV}$ per nucleon. The ${ }^{97} \mathrm{Rh}$ ions following the ${ }^{96} \mathrm{Ru}(\mathrm{p}, \gamma)$ reaction at a cryogenically cooled liquid microjet hydrogen target were detected with Double Sided Silicon Strip Detectors (DSSSD) mounted inside a pocket. We could prove the feasibility of this approach and clearly detected events originating from proton captures on ${ }^{96} \mathrm{Ru}$. The analysis is still in progress and we could therefore only report an upper limit on the cross section at $11 \mathrm{MeV}$, which is slightly above the prediction from the NON-SMOKER code. The detailed analysis will be extended down to 10 and $9 \mathrm{MeV}$. In the future, such experiments have to be performed with particle detectors inside the ultra-high vacuum of the ESR in order to measure in the Gamov window. Additionally a demonstration experiment for $(\alpha, \gamma)$ reactions is planned.

\section{Acknowledgments}

This work is supported by the HGF Young Investigators Projects VH-NG-327 and VH-NG331, the ERC grant no. 203175, HIC for FAIR and F\&E Frankfurt/GSI.

\section{References}

[1] E. Burbidge, G. Burbidge, W. Fowler, F. Hoyle. Rev. Mod. Phys. 29 (1957) 547.

[2] H. Utsunomiya, P. Mohr, A. Zilges, M. Rayet. Nucl. Phys. A 777 (2006) 459.

[3] C. Angulo, M. Arnould, M. Rayet, P. Descouvemont, D. Baye, C. Leclercq-Willain, A. Coc, S. Barhoumi, P. Aguer, C. Rolfs, et al. Nucl. Phys. A 656 (1999) 3.

[4] Q. Zhong, T. Aumann, S. Bishop, K. Blaum, K. Boretzky, et al. ${ }^{96} R u(p, \gamma){ }^{97}$ Rh measurement at the GSI storage ring. Journal of Physics: Conference Series 202 (2010) 012011.

[5] S. Agostinelli, J. Allison, K. Amako, J. Apostolakis, H. Araujo, et al. Nucl. Phys. A 506 (2003) 250.

[6] T. Rauscher, F.-K. Thielemann. Astrophysical Reaction Rates From Statistical Model Calculations. Atomic Data Nucl. Data Tables 75 (2000) 1. 